\title{
A Simplified Organ Donor Model Produced by Permanent Complete Central Nervous System Ischemia in Dogs
}

\author{
Thomas S. Huber and Louis G. D'Alecy
}

The physiologic and pathophysiologic changes of the potential organ donor in the brain-dead state are poorly understood. We have developed a canine model of complete brain death by infusing saline into the subdural space and elevating intracranial pressure above systolic arterial pressure. A snare on the inferior vena cava decreased venous return and limited the Cushing response to 1 to 2 minutes. The electroencephalogram became isoelectric as soon as intracranial pressure was elevated and maintained above systolic arterial pressure. The brain-dead state was confirmed hy several means. No return of electroencephalogram activity was seen throughout the study. Neurologic examination failed to document any cranial nerve or

I MPROVED clinical management of the brain-dead organ donor could impact transplantation twofold. First, the donor pool could potentially be expanded. It has been reported that $20 \%$ of all potential donors are unsuitable or progress to somatic death prior to harvest. ${ }^{2}$ 'I his exacerbates the current shortage of organs; it has been projected that between 10,000 and 15,000 donors are needed to satisfy the current demand ${ }^{3,4}$; however, there are only approximately 2,600 donors per year. ${ }^{4}$ Second, the immediate graft function could potentially be improved; clinical studies have related graft failure to donor hypotension. ${ }^{5-8}$

Our limited knowledge of the brain dead donor and the recommended clinical supports have recently been reviewed..$^{9-11}$ A few basic laboratory studies have recently reported the hemodynamic changes during the evolution to brain death ${ }^{1,11-15}$ and the changes in the hormonal profiles after the induction of brain

From the Departments of Physiology and Surgery, The University of Michigan Medical School, Ann Arbor, MI.

Received May 24, 1990; accepted for publication September 20, 1990

Supported in part by the American Heart Association of Michigan. Dr Huber was a Fellow of the American Heart Association of Michigan and is a recipient of a National Research Service Award.

Address reprint requests to Louis G. D'Alecy, DMD, PhD, Department of Physiology, The University of Michigan Medical School, M7799 Medical Science II, 1301 Catherine St, Ann Arbor, MI 48109-0622.

Copyright $\odot 1991$ by W.B. Saunders Company 0883-9441/91/0601-0002\$05.00/0 spinal cord reflexes after the induction of brain death. Pharmacologic challenges failed to document any baroreceptor function or autonomic cardiovascular control in this state. Blood flow to the central nervous system using the microsphere technique was determined at 1 hour post-brain death. The mean calculated flows were not statistically different than zero. The model provides a simple, controlled, consistent, and relatively noninvasive model of complete brain death. It should facilitate the investigation of the mechanisms leading to somatic death in the brain-dead state and ultimately lead to improved clinical supports.

Copyright $\mathbb{1} 1991$ by W.B. Saunders Company

death. ${ }^{1}$ Acute hormonal insufficiency has been proposed as a mechanistic factor in the degradation of the preparation in both the experimental ${ }^{16,17}$ and clinical settings, ${ }^{18}$ but this has not been supported in all experimental setting $\mathrm{s}^{19,20}$ or in all clinical settings. ${ }^{21-24}$ Species and model variability, the extent of the central nervous system (CNS) insult, and the duration of the Cushing response may account for some of the apparently conflicting reports. We have developed a simplified canine model of complete CNS death to provide a more controlled laboratory setting in which to investigate the mechanisms leading to somatic death in the braindead state.

\section{METHODS AND MATERIALS}

\section{General}

Fifteen fasted adult male mongrel dogs weighing from 15 to $25 \mathrm{~kg}$ were anesthetized with $17.5 \mathrm{mg} / \mathrm{kg}$ IV of thiamylal sodium (Surital; Parke-Davis, Morris Plains, $\mathrm{NJ}$ ) and supplemented as needed until the induction of brain death. The animals were intubated and ventilated (Harvard 607, South Natick, MA) with room air; expired $\mathrm{CO}_{2}$ was continuously monitored (Beckman LB-2, Oxnard, CA) and maintained between $4 \%$ and $5 \%$. Plasma $\mathrm{pH}$ was determined intermittently throughout the experiment (IL $113 \mathrm{pH} / \mathrm{Blood} \mathrm{Gas}$ Analyzer) and normalized with supplemental intravenous sodium bicarbonate $(1.5 \%)$ and ventilator changes as appropriate. An esophageal temperature probe was placed, and body temperature was maintained throughout the protocol at approximately $38^{\circ} \mathrm{C}$ with the aid of external supports: heating pad, blankets, and heat lamps. The urinary bladder was catheterized. Needle electrocardiogram (ECG) leads were placed subcutaneously for continuous recording of limb lead II. The atlanto-occipital membrane was surgically exposed, and two 18-gauge needles were placed through the 
membrane into the cisterna magna; one of these catheters was used for pressurized normal saline infusion and the second for continuous intracranial pressure (ICP) monitoring. Electroencephalogram (EEG) screw electrodes were placed into the skull over the fronto/parietal cortex for continuous recording. The femoral artery was cannulated for continuous measurement of pulsatile and mean arterial pressure (MAP), and it was used as a reference site for the microsphere withdrawal. A Swan-Ganz (SG) catheter (Oximetrix, Mountain View, CA) was advanced from the femoral vein for measurement of pulmonary capillary wedge pressure (PCWP), central venous pressure (CVP), and cardiac output $(\mathrm{CO})$ via the thermodilution method. Normal saline $(1.5 \mathrm{~L})$ was administered through the SG catheter prior to the induction of brain death. An anterolateral thoracotomy was performed through the fifth intercostal space; a 2-0 nylon suture snare was placed around the inferior vena cava (IVC) just caudal to the pericardium. A catheter was placed into the internal mammary artery and advanced into the ascending aorta for the microsphere reference withdrawal. A catheter was placed into the left atrial appendage for the microsphere infusion. Plasma glucose was determined throughout the experiment (YSI Glucose Analyzer, Yellow Springs, $\mathrm{OH}$ ). Cardiac index (CI) was determined by dividing the $\mathrm{CO}$ by the body surface area. Systemic vascular resistance (SVR, dynes $\cdot \mathrm{sec} / \mathrm{cm}^{5}$ \% $\mathrm{m}^{2}$ ) was determined by dividing the difference of the MAP and the CVP by the CI and multiplying by a factor of 80 . After the appropriate hemodynamic control values were recorded, brain death was induced.

\section{Brain Death}

An oscillograph tracing of the induction of brain death is shown in Fig 1. Approximately $7 \mathrm{~mL}$ of freshly drawn, nonheparinized blood was slowly infused into the cisterna magna through one of the 18-gauge needles and allowed to clot for several minutes. Care was exercised during this infusion to prevent the ICP from rising above diastolic arterial pressure and precipitating a Cushing response. The
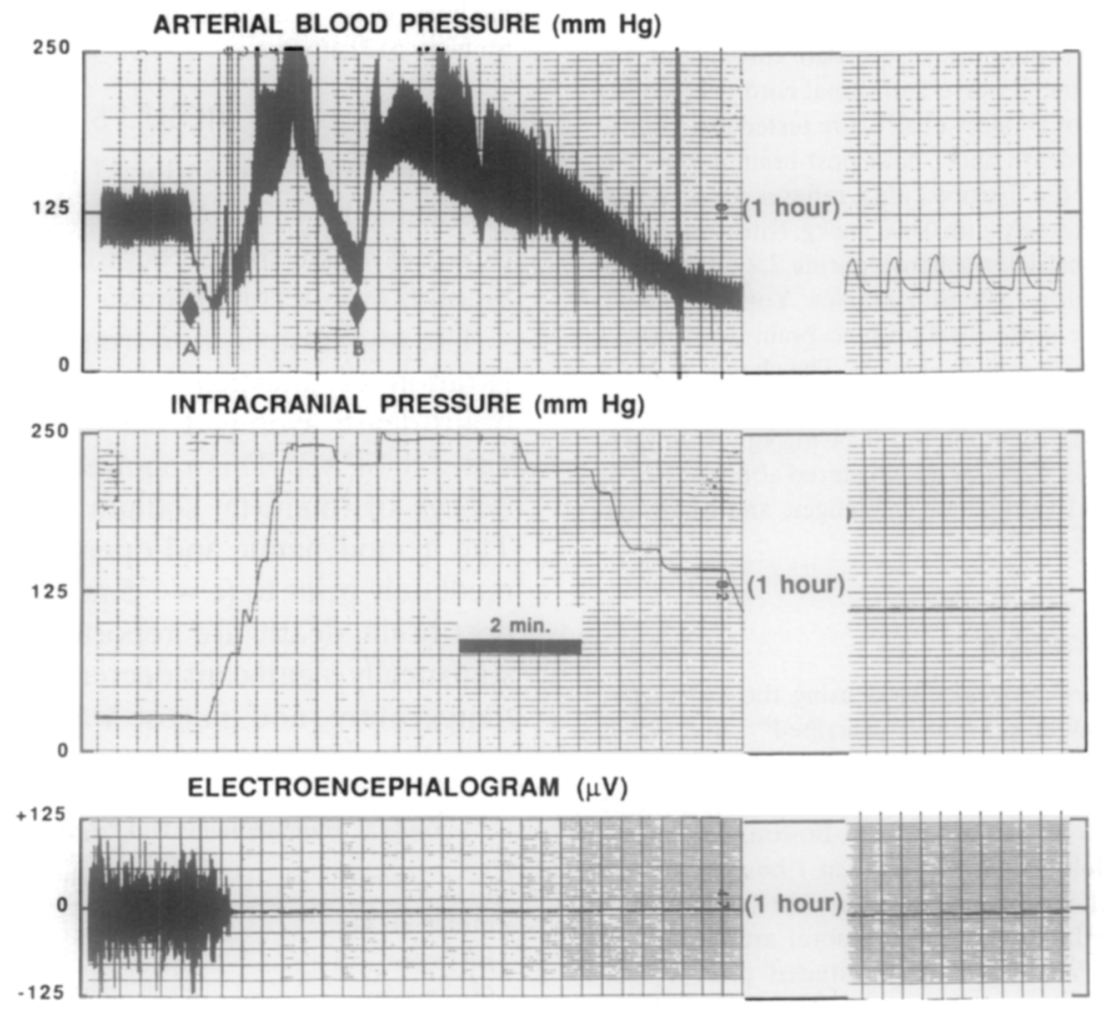

Fig 1. The induction of brain death is shown on an actual oscillograph tracing. Pulsatile arterial pressure and ICP were recorded on a 0 to $250 \mathrm{~mm} \mathrm{Hg}$ scale. EEG was recorded in microvolts on a -125 to 125 scale. The induction was initiated by the withdrawal of the snare on the IVC. This decreased venous return and precipitated a fall in arterial pressure (A). The ICP was raised above systolic arterial pressure by the infusion of saline into the subdural space when it fell below $50 \mathrm{~mm} \mathrm{Hg}$. The ICP was maintained $20 \mathrm{~mm} \mathrm{Hg}$ above the systolic arterial pressure throughout the duration of the study. This rise in ICP caused an almost instantaneous isoelectric EEG and a Cushing response marked by hypertension and bradycardia. The bradycardia cannot be resolved on the oscillograph above because of the slow paper speed. The Cushing response was attenuated by the decrease of venous return caused by the persistent IVC snare and a subsequent fall in arterial pressure resulted (B). The IVC snare was removed when the systolic arterial pressure fell to approximately $50 \mathrm{~mm} \mathrm{Hg}$ the second time. Venous return was augmented and a second hypertensive episode resulted. The mean arterial pressure stabilized between 65 and $75 \mathrm{~mm} \mathrm{Hg}$ within 5 to 10 minutes after the isoelectric EEG. The 1-hour tracing shows the persistently elevated ICP and the isoelectric EEG. No return of electrical activity was seen on the EEG throughout the duration of the study. 
snare on the IVC was then tightened to reduce venous return. When the systolic arterial pressure reached approximately $50 \mathrm{~mm} \mathrm{Hg}$, the ICP was elevated above it and maintained at least $20 \mathrm{~mm} \mathrm{Hg}$ above it throughout the duration of the experiment. ICP was elevated above the systolic arterial pressure within 5 seconds. An isoelectric EEG resulted at this point and brain death was declared. The initial rise in ICP precipitated a marked, but transient, elevation of the arterial pressure from the Cushing reflex. This reflex tends to maintain cerebral perfusion during the elevation of ICP; however, the increase in arterial pressure was limited by the reduced venous return from the snare on the IVC. Within 1 minute, a second fall in the arterial pressure resulted. The snare on the IVC was subsequently removed when the systolic arterial pressure again reached $50 \mathrm{~mm} \mathrm{Hg}$. Venous return was augmented and a second brief rise in the arterial pressure was noted. An initial steady-state condition resulted approximately 5 minutes after the declaration of brain death.

\section{Confirmation of Brain Death}

The EEG was continuously recorded throughout the experiment. The cranial nerve and spinal cord reflexes per the scoring system of D'Alecy et $\mathrm{al}^{25}$ were tested just prior to induction of brain death and 1 hour post-brain death. The responsiveness of the baroreceptor reflexes were tested using vasodilator (nitroglycerin $0.003 \mathrm{mg} / \mathrm{kg}$; Nitrostat, ParkeDavis) and vasoconstrictor (phenylephrine $2.2 \mu \mathrm{g} / \mathrm{kg}$; NeoSynephrine; Winthrop Laboratories, New York, NY) challenges. These were performed prior to brain death and 1 hour after brain death in four animals. The change in MAP and heart rate (HR) were observed. Additionally, a ganglionic blocking agent (pentolinium $0.54 \mathrm{mg} / \mathrm{kg}$; Ansolysen; Wyeth, Philadelphia, PA) was administered after the 1-hour vasoconstrictor and vasodilator challenges, and MAP and HR were recorded.

\section{Microsphere Technique}

Tissue blood flow was calculated using the radioactive microsphere technique previously described ${ }^{26,27}$ and briefly outlined here. Approximately $2 \times 10^{6} 15-\mu \mathrm{m}$ radiolabeled microspheres (randomly chosen tin-113, cerium-141, or scandium-46; New England Nuclear, Boston, MA) were infused into the left atrial appendage at 1 hour post-brain death. Two simultaneous arterial reference samples were withdrawn at $2 \mathrm{~mL} / \mathrm{min}$ from the femoral artery and the ascending aorta. Withdrawal was initiated prior to the microsphere infusion and continued for 2 minutes after the completion. At the conclusion of the experiment, the animals were euthanized with $120 \mathrm{mg} / \mathrm{kg}$ of pentobarbital (Uthol; Butler Co, Columbus, $\mathrm{OH}$ ). An autopsy was performed, and the whole cerebral cortex, cerebellum, brain stem, and spinal cord were harvested. These tissues were sectioned, weighed, desiccated, and counted in the gamma counter (Packard 5330 Gamma Scintillation Spectrometer, Dowmers Grove, IL). The reference blood samples were hemolyzed with concentrated potassium hydroxide, desiccated, and counted in the gamma counter. CNS blood flows were calculated with the reference withdrawal technique.

\section{Experimental Approval}

The experimental procedure conformed to the guidelines established by the American Physiological Society and the National Institutes of Health (Guide for the Care and Use of Laboratory Animals, NIH Publication no. 85-23, 1985), and was approved by The University of Michigan Unit for Laboratory Animal Medicine's Vertebrate Animal Use Committee (approval \#1677A).

\section{Statistical Analyses}

Paired $t$-tests were used to compare the hemodynamic and physiologic variables prior to brain death and 1 hour post-brain death; a level of $P \leq .05$ was accepted as statistically significant. A one-sample $t$-test was used to analyze the calculated microsphere flows and $P \leq .05$ was accepted as statistically significant. The microsphere data analysis was facilitated using the Michigan Interactive Data Analysis System software of The University of Michigan Statistical Research Laboratory (Ann Arbor, MI) on an IBM 3090 computer (New York, NY). All other statistical analyses were performed on a Macintosh II using the Statview 512 software.

\section{RESULTS}

Brain death was induced in 15 animals and confirmed by EEG recording, neurologic exam, pharmacologic baroreceptor challenges, and blood flow determinations.

The total preparation from the initial administration of anesthesia to the declaration of brain death averaged $153 \pm 13$ minutes (values \pm 1 SEM). This required an average total dosage of thiamylal sodium of $37 \pm 3 \mathrm{mg} / \mathrm{kg}$. The hemodynamic and operative variables immediately prior to $(t=-5$ minutes $)$ and 1 hour post-brain death are reported in Table 1. A statistically significant decrease in MAP, SVR, temperature, and glucose was seen after brain

Table 1. Hemodynamic and Physiologic Variables

\begin{tabular}{lcc}
\hline \multicolumn{1}{c}{ Variable } & Pre-Brain Death & 1 h Post-Brain Death \\
\hline MAP $(\mathrm{mm} \mathrm{Hg})$ & $115 \pm 5$ & $61 \pm 4^{*}$ \\
HR $(\mathrm{bpm})$ & $147 \pm 9$ & $131 \pm 3$ \\
$\mathrm{Cl}\left(\mathrm{L} / \mathrm{min} / \mathrm{m}^{2}\right)$ & $4.52 \pm 0.36$ & $4.32 \pm 0.27$ \\
SVR $\left(\mathrm{dynes} \cdot \mathrm{cm}^{5} / \mathrm{m}^{2}\right)$ & $2.107 \pm 136$ & $1,104 \pm 89^{*}$ \\
PCWP $(\mathrm{mm} \mathrm{Hg})$ & $7.1 \pm 0.7$ & $6.9 \pm 0.7$ \\
Temp $\left({ }^{\circ} \mathrm{C}\right)$ & $38.4 \pm 0.2$ & $37.3 \pm 0.2^{*}$ \\
ph $(\mathrm{U})$ & $7.38 \pm 0.02$ & $7.35 \pm 0.02$ \\
Glucose $(\mathrm{mg} / \mathrm{dL})$ & $122 \pm 7$ & $85 \pm 5^{*}$ \\
\hline
\end{tabular}

Note: The hemodynamic and physiologic variables are reported (mean \pm 1 SEM) for the time points immediately prior to brain death ( $t=-5$ minutes) and 1 hour after brain death. The values were compared with a paired $t$-test. Statistical significance was defined as a probability value of $\leq .05$.

*Statistically significant. 
death. There were no significant changes in HR, PCWP, $\mathrm{pH}$, or $\mathrm{Cl}$.

The EEG remained isoelectric throughout the duration of the study. The cranial nerve and spinal cord reflexes were intact prior to the onset of brain death but were absent thereafter. No response to toe pinch, tendon stretch, pupillary light stimuli, etc, was observed in any animal after brain death was induced.

The pressor challenge prior to brain death increased MAP by $14 \pm 3 \mathrm{~mm} \mathrm{Hg}$ and decreased HR $12 \pm 2$ beats per minute (bpm); post-brain death MAP increased $25 \pm 6 \mathrm{~mm}$ $\mathrm{Hg}$, but no change in $\mathrm{HR}$ was detected. The ability to respond to a pressor challenge with a decrease in HR was lost in the brain-dead state despite a greater increase in MAP. Depressor challenges prior to brain death decreased MAP $22 \pm 3 \mathrm{~mm} \mathrm{Hg}$ and increased HR $12 \pm 3 \mathrm{bpm}$; post-brain death MAP decreased $23 \pm 6 \mathrm{~mm} \mathrm{Hg}$ and no rise in HR was detected. Similarly, the brain-dead state lost the ability to respond to the depressor challenge with an increase in the heart rate. In the brain-dead preparation, ganglionic blockade caused no detectable change in HR or MAP. Ganglionic blockade in two shamoperated non-brain-dead dogs produced a mean 35-mm $\mathrm{Hg}$ decrease in MAP and a mean 42bpm increase in $\mathrm{HR}$.

Calculated blood flow values obtained with the microsphere technique at the 1 hour point after the induction of brain death are presented in Table 2. All the brain and spinal cord tissues were counted to obtain these values. The mean calculated flow for 20 tissues in 15 dogs was found to be $-0.05 \mathrm{~mL} / \mathrm{min} / 100 \mathrm{~g}$. This was not statistically different from zero with a one- sample $t$-test. The negative flow value reflects correction to the background counts. Eighteen of 20 individual samples were not statistically different from zero. The highest $(0.45 \mathrm{~mL} / \mathrm{min} /$ $100 \mathrm{~g})$ and lowest $(-0.31 \mathrm{~mL} / \mathrm{min} / 100 \mathrm{~g})$ calculated blood flow values should be considered zero because they are within the error of the microsphere technique.

\section{DISCUSSION}

There have been a variety of experimental models used to approximate the clinical setting and investigate the brain-dead state. Novitzky et $\mathrm{al}^{1}$ and Lin et $\mathrm{al}^{28}$ have reported studies in baboons and dogs, respectively, in which brain death was precipitated by the inflation of a balloon in the intracranial cavity. Wicomb et $\mathrm{al}^{1.3}$ and Finkelstein et al $^{19}$ have reported pig and dog studies, respectively, in which the state was produced by serial ligation of the vascular supply to the brain. Blaine et $\mathrm{al}^{29}$ used a pig model in which the total cranial contents were evacuated, and Cowley et $\mathrm{al}^{30}$ reported studies in which dogs were decapitated. Each of these models represents a substantially greater surgical manipulation and associated trauma than the model described. With the simplicity of our preparation, a more consistent model of complete brain death is provided for the assessment of metabolic, endocrine, and hemodynamic changes associated with this process.

The variety of species, mechanism of producing brain death, and documentation of the extent of CNS involvement have limited the conclusions and comparisons among the individual experimental studies. The duration of the induced Cushing responses has varied in pub-

Table 2. CNS Blood Flow

\begin{tabular}{lllr}
\hline Brain & & & $-0.18 \pm 0.09$ \\
R frontal & $-0.31 \pm 0.17$ & L frontal & $-0.23 \pm 0.01$ \\
R occipital & $-0.26 \pm 0.16$ & L occipital & $-0.21 \pm 0.10$ \\
R temporal & $-0.23 \pm 0.14$ & L temporal & $-0.20 \pm 0.16$ \\
R anterior parietal & $-0.08 \pm 0.15$ & L anterior parietal & $0.02 \pm 0.21$ \\
R post parietal & $-0.12 \pm 0.11$ & L post parietal & $-0.04 \pm 0.13$ \\
R thalamus & $-0.19 \pm 0.16$ & L Thalamus & $-0.00 \pm 0.09$ \\
Cerebellum & $-0.06 \pm 0.08$ & Brain stem & $0.07 \pm 0.07$ \\
Spinal cord & & & $0.09 \pm 0.03$ \\
Cervical cephalad & $0.23 \pm 0.13$ & Cervical caudad & $0.45 \pm 0.17$ \\
Thoracic cephalad & $0.10 \pm 0.11$ & Thoracic caudad & Sacral \\
Lumbar & $0.10 \pm 0.05$ & . &
\end{tabular}

Note: The microsphere calculated blood flows (mean \pm 1 SEM) at 1 hour post-brain death are expressed in $\mathrm{mL} / \mathrm{min} / 100 \mathrm{~g}$ of tissue. All of the brain and spinal cord tissues were harvested, and the values are reported by their anatomic location. 
lished studies from 20 to 135 minutes, ${ }^{1,13}$ with some reports indicating myocardial ischemic changes on ECG and histologic examination. ${ }^{1,12,14,15}$ These responses could potentially contribute in a variable manner to the deterioration of the preparation. The induction of brain death in our model was intentionally brief and the Cushing response was limited to approximately 1 to 2 minutes. A stable blood pressure in the brain-dead state was produced within 5 to 10 minutes of the elcvation of the ICP.

The clinical diagnosis of brain death requires the absence of the cranial nerve reflexes without exogenous CNS depression and an appropriate period of observation. Simple adherence to these clinical criteria for brain death is not useful in the laboratory and makes comparison of results from different studies unwarranted. The presence or absence of the medullary cardiovascular center has a profound impact on the hemodynamic stability of either the clinical donor or the experimental preparation. Mechanistic studies of the deterioration in the braindead state need to consistently report the functional integrity of this center to allow any generalizations of the data. Our model unequivocally eliminates the function of this center.

Although the present model is consistent and reproducible, it does not replicate all clinical settings; paradoxically, several of the experimental advantages account for the limitations of immediate clinical relevance. The Cushing response may be a mechanistic component of the deterioration of the individual progressing toward the brain-dead state. The basic model as presented limited this component and presumably eliminated its longer-term effects. However, subsequent studies can build on these baseline observations and examine, as a controlled experimental variable, the duration and extent of the Cushing response in this setting. Furthermore, the preparation lacks spinal cord blood flow and spinal cord function as assessed by neurologic exam. While this oversimplifies many clinical situations, spinal cord reflexes have been reported absent in up to $25 \%$ of brain-dead patients by Ivan. ${ }^{31}$ The contribution of the spinal cord to the maintenance of homeostasis and cardiovascular stability in the braindead state, while potentially important, is cur- rently undefined and requires a controlled model for full quantification. A final simplification is that while the animal is subjected to an operative procedure, there are minimal additional traumatic injuries. We feel that these simplifications do impose immediate limitations of clinical relevance, but the simplicity and reproducibility of the model allow for future controlled studies of these clinical complications.

Application of the present model should elucidate the optimal clinical management of the potential organ donor. The optimal volume support regimen has not been determined. Currently, volume support is a balance between the need to maintain adequate renal perfusion and urine output against the development of interstitial and end-organ edema. Similarly, the role of pressors and the optimal agent in this setting have not been determined. The standard pressor agents likely have different effects in the absence of an intact CNS. This is particularly relevant to the distribution of cardiac output and tissue perfusion. Additionally, the alteration of the plasma hormonal profiles has not been consistently described and the efficacy of hormonal supplementation has not been resolved.

Several other aspects of the model merit discussion. Thiamylal sodium was selected as a general anesthetic agent because it is an ultrashort-acting barbiturate. No additional anesthetic agent was required after the animals became brain dead, and we presumed that the agent was cleared and exerted minimal effects on this state. Once blood flow to the CNS was stopped, the thiamylal sodium would presumably no longer exert its effects. In our preliminary studies, we were unable to overcome the reflex hypertension by simply raising the ICP, as reported previously by Lanier et al. ${ }^{32}$ The snare was placed around the inferior vena cava to allow us to overcome the Cushing response. Vasodilators were tried and afforded a similar ability to overcome the Cushing response, but their effects persisted long after the induction of brain death and unpredictably destabilized the preparation. The blood that was infused into the subdural space prior to the initiation of brain death facilitated the maintenance of ICP above MAP with a minimal requirement for 
saline infusion. We presume that the blood blocks the arachanoid granules and prevents the outflow from CSF. Minimal myocardial irritability was seen during the induction of brain death, and normal sinus rhythm persisted through the 4-hour observation period. These findings contrast with other investigators who have documented extensive ECG changes generally in association with a prolonged Cushing response. . $^{.12-15}$

This is the first time that the absence of blood flow to the CNS has been definitively documented with the microsphere technique in a model of the potential organ donor. Ljunggren et al, ${ }^{3.3}$ using rats, and Neely and Youmans ${ }^{34}$ and Lanier et $\mathrm{al}^{32}{ }^{32}$ using dogs, have investigated neurologic outcome following compression ischemia or "bloodless" cerebral ischemia. They elevated ICP above arterial pressure by subdural infusion for various durations then normalized ICP to permit cerebral reperfusion. Cerebral blood flow was documented in one animal by Neely and Youmans ${ }^{34}$ using an injection of radioactive ${ }^{24} \mathrm{Na}$ and performing brain biopsies; no activity in the brain was seen at 15 minutes postelevation of ICP above arterial pressure. Lanier et $\mathrm{al}^{32}$ injected indium-labeled red blood cells in two animals and showed that the cerebral blood volume was $12 \%$ to $14 \%$ of control values after the elevation of the ICP above arterial pressure. Kramer and Tuynman ${ }^{35}$ used a similar model in dogs and cats to investigate the state of cerebral deanimation or "coma depasse"; ICP was raised above arterial pressure for various intervals and the subsequent hemodynamic changes reported. They performed angiograms on two animals when the ICP exceeded arterial pressure and reported the absence of cerebral blood flow. Our documentation of CNS blood flow with microspheres included all of the brain and spinal cord and illustrated the absence of blood flow to these tissues in all animals.

This model of total brain death in the dog is simple and relatively noninvasive. Brain death was documented by the absence of an EEG. neurologic examination, the absence of baroreceptor function, and the absence of blood flow to the CNS. The clinical limitations of the model have been recognized, but the control, simplicity, and reproducibility of the preparation should facilitate the investigation of the mechanistic questions leading to somatic death in the brain-dead state and lead ultimately to a rational approach to optimizing clinical support of the organ donor.

\section{ACKNOWLEDGMENT}

The authors would like to sincerely thank Susan Stante, Brad Martin, and Paddy O'Halloran for their excellent technical assistance.

\section{REFERENCES}

1. Novitzky D, Wicomb WN, Cooper DKC, et al: Electrocardiographic, hemodynamic, and endocrine changes occurring during experimental brain death in the chacma baboon. Heart Transplant 4:63-69, 1984

2. Jenkins AM: Causes of failure to harvest cadaver kidneys for transplantation. Br Med J 1:816-817, 1976

3. Stuart FP: Need, supply and legal issues related to organ transplantation in the United States. Transplant Proc 16:87-94, 1984

4. Evans RW, Manninen DL, Garrison LP, et al: Donor availability as the primary determinant of the future of heart transplantation. JAMA 255:1892-1898, 1986

5. Schneider A, Toledo-Pereyra LH, Zeichner WD, et al: Effects of dopamine and pitressin on kidneys procured and harvested for transplantation. Transplant Proc 36:110, 1983

6. Toledo-Pereyra LH, Callender CO, Moberg AW, et al: Clinical effects of warm ischemia and prolonged preservation on the outcome of transplanted kidneys. Minn Med $59: 18,1976$

7. Toledo-Pereyra LH, Moberg AW, Callender CO, et al:
Factors determining early kidney function following clinical preservation. Minn Med 58:446, 1975

8. Santiago-Delpin EA, Toledo-Pereyra LH, Moberg AW, et al: Predictive parameters for renal preservation. Trans Am Soc Artif Intern Organs 20:550, 1974

9. Soifer BE, Gelb AW: The multiple organ donor: identification and management. Ann Intern Med 110:814 823,1989

10. Darby IM, Stein K, Grenvik A, et al: Approach to management of the heartbeating "brain dead" organ donor. JAMA 261:2223-2228, 1989

11. Turcotte JG: Conventional management of the braindead potential multi-organ donor. Transplant Proc 20:5-8. 1988 (suppl 7)

12. Novitzky D, Wicomb WN, Rose AG, et al: Pathophysiology of pulmonary edema following experimental brain death in the chacma baboon. Ann Thorac Surg 43:288-294, 1987

13. Wicomb WN, Cooper DKC, Lanza RP, et al: The effects of brain death and 24 hours' storage by hypothermic 
perfusion on donor heart function in the pig. $J$ Thorac Cardiovasc Surg 91:896-909, 1986

14. Novitzky D, Wicomb WN, Cooper DKC, et al: Prevention of myocardial injury during brain death by total cardiac sympathectomy in the chacma baboon. Ann Thorac Surg 41:520-524, 1986

15. Novitzky D, Cooper DKC, Rose AG, et al: Prevention of myocardial injury by pretreatment with verapamil hydrochloride prior to experimental brain death: Efficacy in a baboon model. Am J Emerg Med 5:11-18, 1987

16. Novitzky D, Cooper DKC, Morrell D, et al: Change from aerobic to anaerobic metabolism after brain death, and reversal following triiodothyronine therapy. Transplantation 45:32-36, 1986

17. Novitzky D, Wicomb WN, Cooper DKC, et al: Improved cardiac function following hormonal therapy in brain dead pigs: relevance to organ donation. Cryobiology 24:1-10, 1987

18. Novitzky D, Cooper DKC, Reichart B: Hemodynamic and metabolic responses to hormonal therapy in brain dead potential organ donors. Transplantation 43:854, 1987

19. Finkelstein I, Toledo-Pereyra LH, Castellanos J: Physiologic and hormonal changes in expcrimentally induced brain dead dogs. Transplant Proc 19:4156-4158, 1987

20. Castellanos J, Toledo-Pereyra LH, Finkelstein I: Hormonal response to experimentally induced brain death. Transplant Proc 20:731-732, 1988

21. Macoviak JA, McDougall IR, Bayer MF, et al: Significance of thyroid dysfunction in human cardiac allograft procurement. Transplantation 43:824-826, 1987

22. Wahlers T, Fieguth HG, Jurmann $M$, et al: Does hormone depletion of organ donors impair myocardial function after cardiac transplantation? Transplant Proc 20:792-794, 1988 (suppl 1)

23. Keogh AM, Howlett TA, Perry L, et al: Pituitary function in brain-stem dead organ donors: A prospective survey. Transplant Proc 20:729-730, 1988
24. Robertson KM, Hramiak IM, Gelb AW: Endocrine changes and haemodynamic stability after brain death. Transplant Proc 21:1197-1198, 1989

25. D'Alecy LG, Lundy EF, Barton KJ, et al: Dextrose containing intravenous fluid impairs outcome and increases death after eight minutes of cardiac arrest and resuscitation in dogs. Surgery 100:505-511, 1986

26. D'Alecy LG, Rose CJ, Sellers SA: Sympathetic modulation of hypercapnic cerebral vasodilation in dogs. Circ Res 45:771-785, 1979

27. Van Wylen DGL, D'Alecy LG: Regional blood flow distribution during the Cushing response: Alterations with adrenergic blockade. Am J Physiol 248:H98-H108, 1985

28. Lin $\mathrm{H}$, Okamoto $\mathrm{R}$, Yamamoto $\mathrm{Y}$, et al: Hepatic tolerance to hypotension as assessed by the changes in arterial ketone body ratio in the state of brain death. Transplantation 47:444-448, 1989

29. Blaine EM, Tallman RD, Frolicher D, et al: Vasopressin supplementation in a porcine model of brain-dead potential organ donors. Transplant Proc 38:459-464, 1984

30. Cowley AW, Monos E, Guyton AC: Interaction of vasopressin and the baroreceptor reflex system in the regulation of arterial blood pressure in the dog. Circ Res 34:505-514, 1974

31. Ivan LP: Spinal reflexes in cerebral death. Neurology 23:650-652, 1973

32. Lanier WL, Fleischer JE, Milde JH, et al: Postischemic neurologic recovery and cerebral blood flow using a compression model of complete "bloodless" cerebral ischemia in dogs. Resuscitation 16:271-286, 1988

33. Ljunggren B, Ratcheson RA, Siesjo BK: Cerebral metabolic state following complete compression ischemia. Brain Res 73:291-307, 1974

34. Neely WA, Youmans JR: Anoxia of canine brain without damage. JAMA 183:93-95, 1963

35. Kramer A, Tuynman JA: Acute intracranial hypertension-an experimental investigation. Brain Res 6:686-705, 1967 